\title{
Pneumatically Driven Vertical Articulated Robotic Arm for Surgical Task with Inertia Estimation
}

\author{
Takuya Iwai, ${ }^{1 *}$ Tetsuro Miyazaki, ${ }^{2}$ Toshihiro Kawase, ${ }^{1,3}$ \\ Takahiro Kanno, ${ }^{4}$ and Kenji Kawashima ${ }^{2}$ \\ ${ }^{1}$ Institute of Biomaterials and Bioengineering, Tokyo Medical and Dental University, \\ 2-3-10 Kandasurugadai, Chiyoda-ku, Tokyo 101-0062, Japan \\ ${ }^{2}$ Department of Information Physics and Computing, Tokyo University, \\ 7-3-1 Hongo, Bunkyo-ku, Tokyo 113-8656, Japan \\ ${ }^{3}$ Institute of Innovative Research, Tokyo Institute of Technology, \\ 4259, Midori-ku, Yokohama, Kanagawa 226-8503, Japan \\ ${ }^{4}$ Riverfield Inc, Yotsuya Medical Bldg. 5th floor, 20 Samon-cho, Shinjuku-ku, Tokyo, Japan
}

(Received October 30, 2020; accepted January 14, 2021)

Keywords: surgical robot, robotic arm, pneumatic system, inertia estimation

In this study, we developed a vertical articulated robotic arm for laparoscopic surgery and proposed a method of controlling the robot. The robotic arm operates surgical instruments around a virtually fixed remote center of motion (RCM) calculated from the robot kinematics. The robotic arm has six degrees of freedom and is driven by pneumatic actuators. The features of the pneumatic actuators of compactness, high backdrivability, and low heat generation allow the robotic arm to be moved passively by the human hand and prevent the risk of heat accumulation in the drape. We made the upper arm and forearm lightweight by mounting the pneumatic actuators on the base of the robot. The joint angles are controlled by pneumatic servo systems. The inertia of the forearm and upper arm was estimated by measuring the joint angles. We also proposed variable gain control of the yaw joint to compensate for changes in inertia. We experimentally confirmed that the variable gain improves the controllability of the robot and improves its operability around the virtual fixed RCM.

\section{Introduction}

In laparoscopic surgery, a master-slave-type surgical robot system is widely used for surgical operations. ${ }^{(1-8)}$ A surgical robot consists of several robotic arms equipped with surgical instruments such as a laparoscope and forceps. A robotic arm has multiple joints for pivoting instruments around a remote center of motion (RCM) and inserting them into the abdominal cavity. The commercially available surgical robot da Vinci has an RCM mechanism that achieves pivot motion through a mechanical linkage. ${ }^{(4)}$ The fixed RCM provides precise pivot motion. However, before the surgical operation, the operator must adjust the robot manually until the RCM of the robot coincides exactly with the pivot. Robotic arms are usually driven

*Corresponding author: e-mail: tiwai.bmc@tmd.ac.jp

https://doi.org/10.18494/SAM.2021.3153 
by electric motors with a reduction mechanism. ${ }^{(9-16)}$ The electrically driven system enables precise motion control. However, a force sensor must be attached to the end effector to detect manual operation, which makes robotic arms expensive. This problem can be avoided if direct drive motors without reduction gears drive the joints. However, such motors tend to be large. In addition, a large current is required to generate a large joint torque, which causes the motor to generate heat. Heat generation must be prevented in a surgical robot, especially the robotic arm, since it is covered by a drape to protect against infection. A robotic arm that is driven by pneumatic actuators can solve these problems. A pneumatic actuator generates a high driving force without a reduction mechanism. It has high backdrivability and almost no heat generation. ${ }^{(17-20)}$ Moreover, a pneumatically driven robotic arm can be moved passively by a human hand. Tadano et al. developed a lightweight and compact pneumatically driven robot for laparoscopic surgery. ${ }^{(19)}$ The robot has a mechanically fixed RCM similar to da Vinci. The mechanism of the fixed RCM needs as few as three joints, making the mechanism simple. However, the fixed RCM needs to be adjusted to the pivot point, which is time-consuming. The surgical robot named DLR MIRO uses a vertical articulated robotic arm instead of a fixed RCM. The robot is driven by electric motors $^{(16)}$ with surgical instruments installed at the tip of the robotic arm. The robotic arm inserts the surgical instruments into the abdominal cavity and operates them around the RCM. The RCM is not fixed mechanically, and motion around the virtual RCM is achieved by calculating the pivot from a kinematic model, making the adjustment of the robot to the pivot easier. However, force sensors must be attached to the arm for manual operation. The mass of the robotic arm is $10 \mathrm{~kg}$ and mass reduction is desired.

In this study, a pneumatically driven robotic arm for laparoscopic surgery was developed. Figure 1 shows the developed robotic arm, which is a vertical articulated robotic arm like a

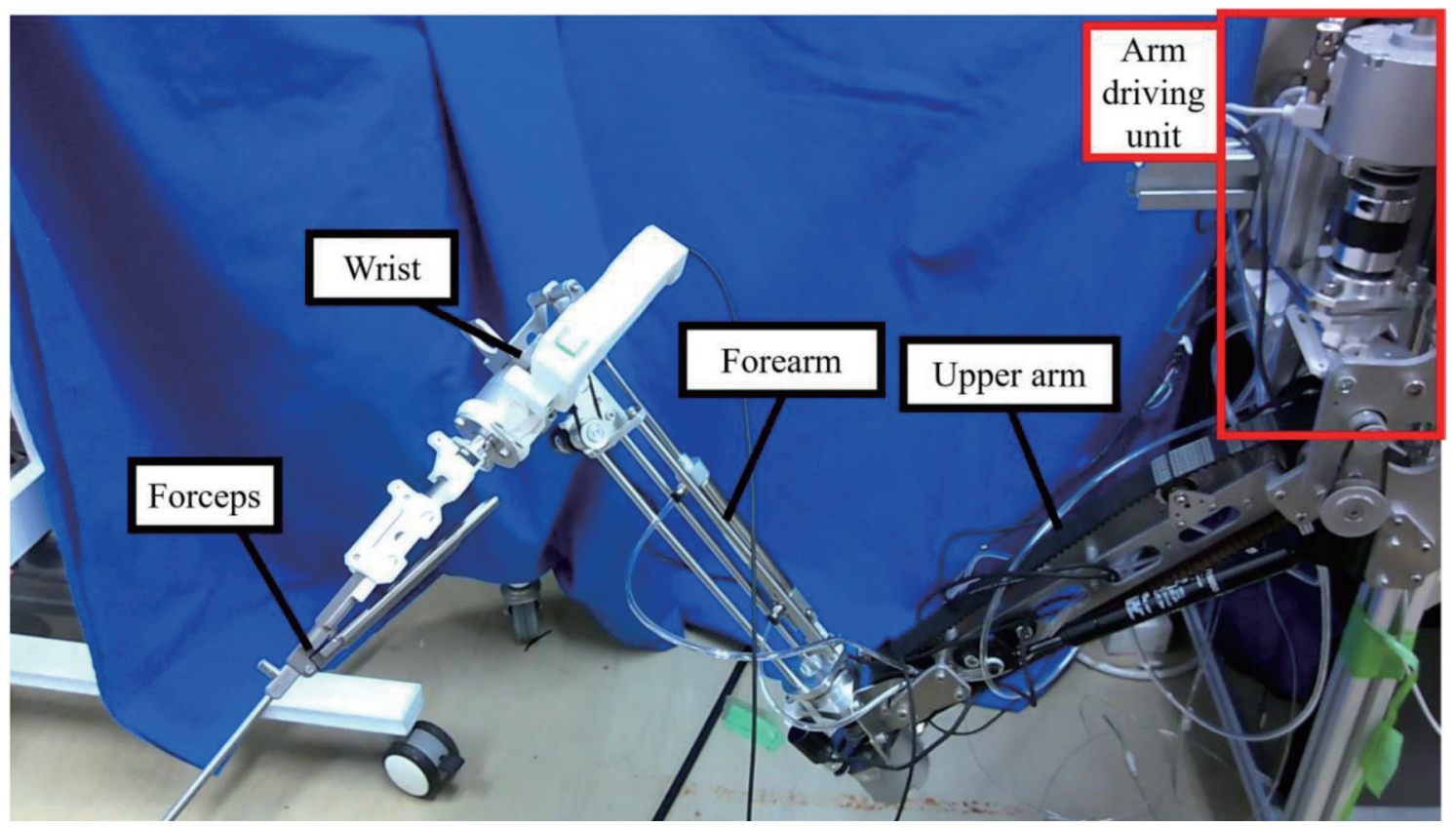

Fig. 1. (Color online) Pneumatically driven robotic arm. 
human arm. The pneumatic actuators are lightweight and compact compared with electric motors, reducing the mass of the robotic arm to about $4 \mathrm{~kg}$. The instrument can be installed on an end effector. The virtual fixed RCM is estimated from the kinematic model of the robotic arm.

This paper is organized as follows. In Sect. 2, the mechanical design of the robotic arm is presented. In Sect. 3, a pneumatic control system and variable gain control to decrease the effect of the inertia of the arm are proposed. The effect of the variable gain is evaluated, and experiments in which the robotic arm moves forceps via pivoting are reported in Sect. 4. Finally, the conclusion is presented in Sect. 5.

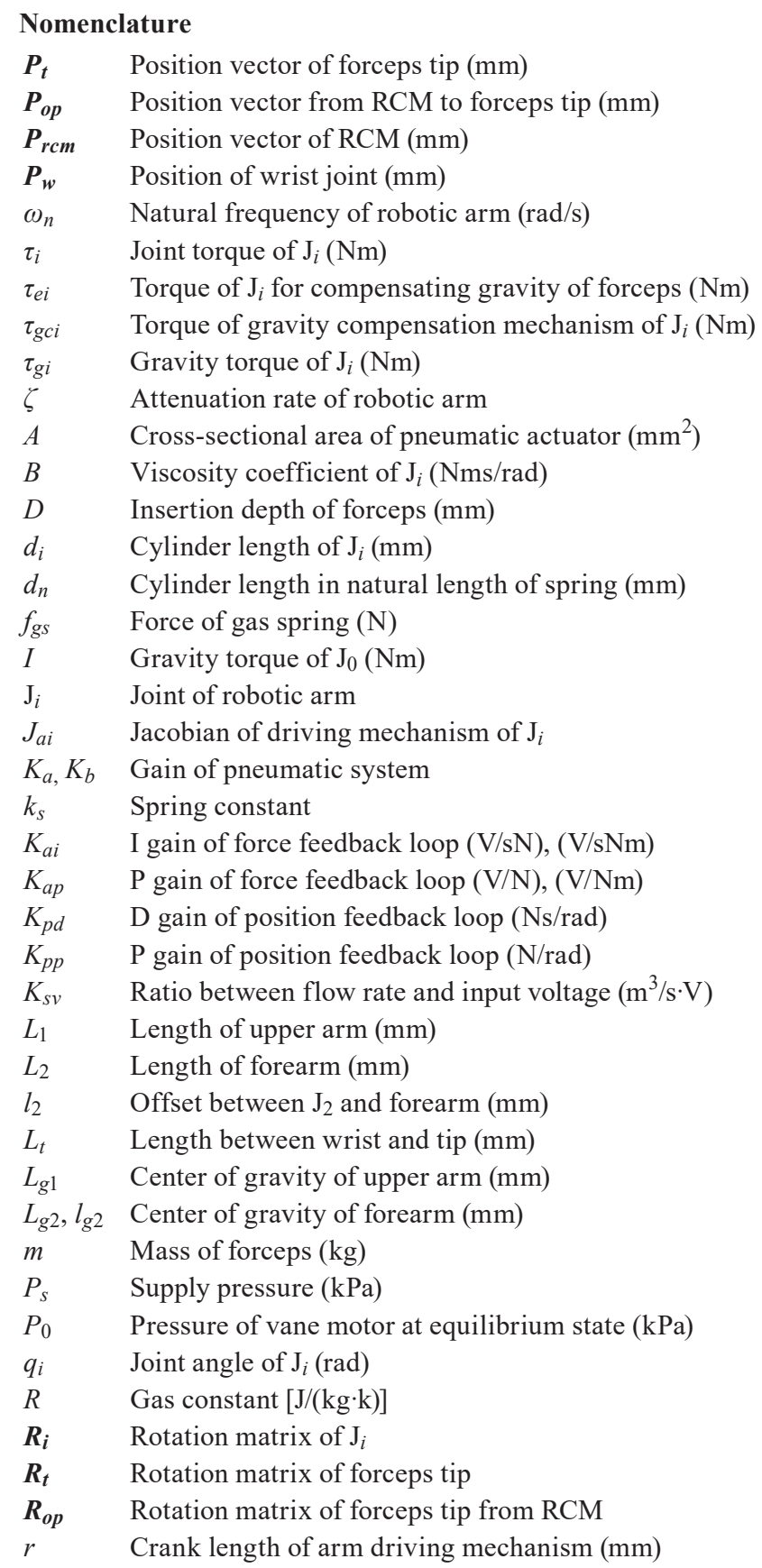




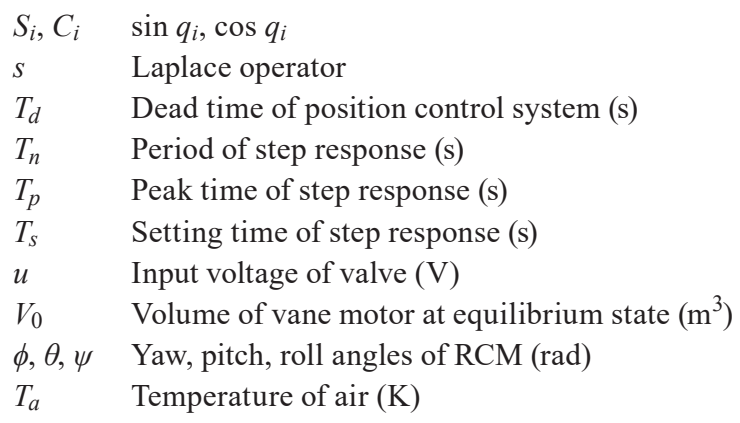

\section{Mechanical Design}

\subsection{Robotic arm}

The robotic arm consists of an upper arm, a forearm, and a wrist, like a human arm. The upper arm and forearm are driven by an arm driving unit. Figure 2 shows the mechanism of the robotic arm. We define the origin of a static $x-y-z$ coordinate system on the root of the robotic arm. The wrist is driven by actuators placed in the forearm. The robotic arm has six rotational joints. The payload at the wrist joint to move the surgical instruments is $1 \mathrm{~kg}$. The pneumatic actuators for driving the upper arm and forearm are placed on the root of the robotic arm, making the upper arm and forearm lightweight and compact. The rotation of the forearm is transmitted to the arm driving unit via a timing pulley mechanism. The timing pulley mechanism decreases the torque of $J_{1}$ to compensate for the gravity force of the forearm and the instruments. The gravity force exerted on the forearm generates the torques $\tau_{g 1}+\tau_{g 2}$ and $\tau_{g 2}$, which lower the upper arm and forearm, respectively. The torques of the upper arm and forearm rotate the pulley clockwise and counterclockwise, respectively. Thus, the pulley mechanism

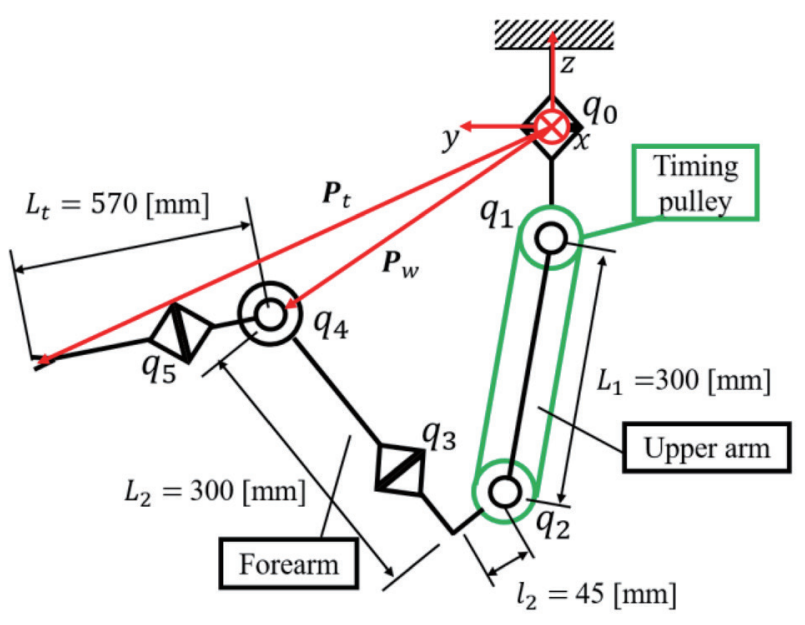

Fig. 2. (Color online) Mechanism of the robotic arm. 
cancels the torque $\tau_{g 2}$, and the torque $\tau_{g 1}$ is simply added to joint $\mathrm{J}_{1}$. The torque $\tau_{g 1}$ is equivalent to that when the force is exerted on the tip of the upper arm. This mechanism allows the actuators to be mounted on the root of the robotic arm instead of on the inside of the forearm, decreasing the torque $\tau_{1}$ generated by the mass of the object on the end effector. The forward kinematics is given as follows:

$$
\begin{gathered}
\boldsymbol{P}_{t}=\boldsymbol{R}_{\mathbf{0}}\left(\boldsymbol{R}_{\mathbf{1}}\left(\left[\begin{array}{c}
0 \\
L_{1} \\
0
\end{array}\right]+\boldsymbol{R}_{\mathbf{2}}\left(\left[\begin{array}{c}
0 \\
L_{2} \\
-l_{2}
\end{array}\right]+\boldsymbol{R}_{\mathbf{3}} \boldsymbol{R}_{\mathbf{4}}\left[\begin{array}{c}
0 \\
L_{t} \\
0
\end{array}\right]\right)\right)\right), \\
\boldsymbol{R}_{\boldsymbol{t}}=\boldsymbol{R}_{0} \boldsymbol{R}_{2} \boldsymbol{R}_{3} \boldsymbol{R}_{4} \boldsymbol{R}_{5},
\end{gathered}
$$

where $\boldsymbol{P}_{\boldsymbol{t}}$ is the position vector between the root of the robotic arm and the end effector. $\boldsymbol{R}_{\boldsymbol{t}}$ is the rotation matrix of the end effector. Joint angle $q_{1}$ does not affect the rotation matrix $\boldsymbol{R}_{\boldsymbol{t}}$ because of the timing pulley.

To obtain the reference joint angles, the inverse kinematics must be calculated. The inverse kinematics is given by the following steps:

1. The position vector $\boldsymbol{P}_{\boldsymbol{w}}$ between the root of the robotic arm and the wrist joint is calculated using Eq. (3), Then, joint angle $q_{0}$ is obtained using Eq. (4).

$$
\begin{gathered}
\boldsymbol{P}_{w}=\left[\begin{array}{l}
x_{w} \\
y_{w} \\
z_{w}
\end{array}\right]=\boldsymbol{P}_{\boldsymbol{t}}-\boldsymbol{R}_{\boldsymbol{t}}\left[\begin{array}{c}
0 \\
L_{t} \\
0
\end{array}\right] \\
q_{0}=\tan ^{-1}\left(\frac{x_{w}}{y_{w}}\right)
\end{gathered}
$$

2. The position of the wrist joint on the plane of the arm motion space is calculated using Eq. (5). Then, joint angles $q_{1}$ and $q_{2}$ are obtained by minimizing the position error $\boldsymbol{E}_{\boldsymbol{r e f}}$ given by Eq. (6).

$$
\begin{gathered}
\boldsymbol{P}_{w}^{\prime}=\left[\begin{array}{c}
x_{w}^{\prime} \\
y_{w}^{\prime} \\
z_{w}^{\prime}
\end{array}\right]=\boldsymbol{R}_{0}^{t} \boldsymbol{P}_{w} \\
\boldsymbol{E}_{\text {ref }}=\left(\left[\begin{array}{c}
y_{w}^{\prime} \\
z_{w}^{\prime}
\end{array}\right]-\left[\begin{array}{l}
L_{1} C_{1}+L_{2} C_{1}+l_{2} S_{2} \\
L_{1} S_{1}+L_{2} S_{1}+l_{2} C_{2}
\end{array}\right]\right)
\end{gathered}
$$

3. The wrist joint angles $q_{3}$ and $q_{5}$ are given by Eq. (7) using the wrist rotational matrix $\boldsymbol{R}_{\boldsymbol{w}}$ derived from Eq. (8). 


$$
\begin{gathered}
{\left[\begin{array}{l}
q_{3} \\
q_{5}
\end{array}\right]=\left[\begin{array}{c}
\tan ^{-1}\left(\frac{\boldsymbol{R}_{w}(1,2)}{\boldsymbol{R}_{w}(3,2)}\right) \\
\tan ^{-1}\left(\frac{\boldsymbol{R}_{w}(2,1)}{\boldsymbol{R}_{w}(2,3)}\right)
\end{array}\right]} \\
\boldsymbol{R}_{\boldsymbol{w}}=\boldsymbol{R}_{2}^{\boldsymbol{t}} \boldsymbol{R}_{0}{ }_{0} \boldsymbol{R}_{\boldsymbol{t}}
\end{gathered}
$$

Here, $\boldsymbol{R}_{\boldsymbol{w}}(i, j)$ is the $i$ th row and $j$ th column element of $\boldsymbol{R}_{\boldsymbol{w}}$.

4. Joint angle $q_{4}$ is given by Eqs. (9) and (10).

$$
\begin{gathered}
q_{4}=\tan ^{-1}\left(\frac{z_{t}^{\prime}}{y_{t}^{\prime}}\right) \\
\boldsymbol{P}_{\boldsymbol{t}}^{\prime}=\left[\begin{array}{c}
x_{t}^{\prime} \\
y_{t}^{\prime} \\
z_{t}^{\prime}
\end{array}\right]=\boldsymbol{R}_{3}^{t} \boldsymbol{R}_{\boldsymbol{w}}\left[\begin{array}{l}
0 \\
1 \\
0
\end{array}\right]
\end{gathered}
$$

\subsection{Driving mechanism}

The arm driving unit, shown in Fig. 3, has a yaw joint and two pitch joints. A vane motor actuates the yaw joint $\mathrm{J}_{0}$. The pneumatic cylinders (SMC, CJ2XD16-60Z) that actuate the two pitch joints $J_{1}$ and $J_{2}$ are arranged around the vane motor. The pneumatic cylinders drive joints $J_{1}$ and $J_{2}$ through the slider-crank mechanism shown in Fig. 4 . The relationship between the joint torque and the driving force of the cylinder is

$$
\tau_{i}=\frac{r\left\{(L+l) S_{i}-(L+l) C_{i}\right\}}{d_{i}} f_{i}=J_{a i} f_{i} .
$$

Equation (11) is used to obtain the reference driving force from the reference joint torque. The gravity torque generated by the mass of the upper arm and forearm lowers the arms. The gravity torque of joints $\mathrm{J}_{1}$ and $\mathrm{J}_{2}$ is

$$
\tau_{g}=\left[\begin{array}{c}
\tau_{\mathrm{g} 1} \\
\tau_{\mathrm{g} 2}
\end{array}\right]=\left[\begin{array}{c}
\left(M_{1} L_{\mathrm{g} 1}+M_{2} L_{1}\right) g \mathrm{C}_{1} \\
M_{2} g\left(L_{\mathrm{g} 2} \mathrm{C}_{2}+l_{\mathrm{g} 2} \mathrm{~S}_{2}\right)
\end{array}\right] .
$$

Increasing the number of pneumatic cylinders to overcome the torque $\tau_{g}$ would increase the size of the driving unit and the flow rate. Therefore, a gravity compensation mechanism is 


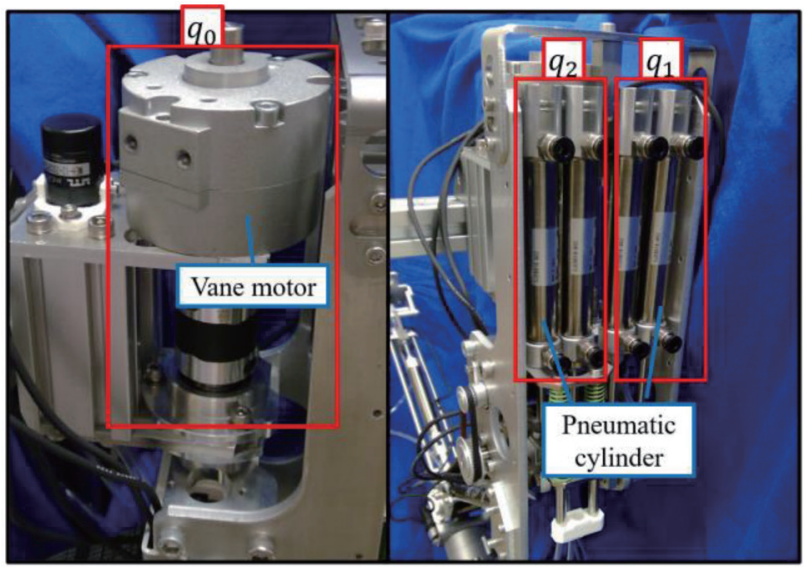

Fig. 3. (Color online) Arm driving unit.

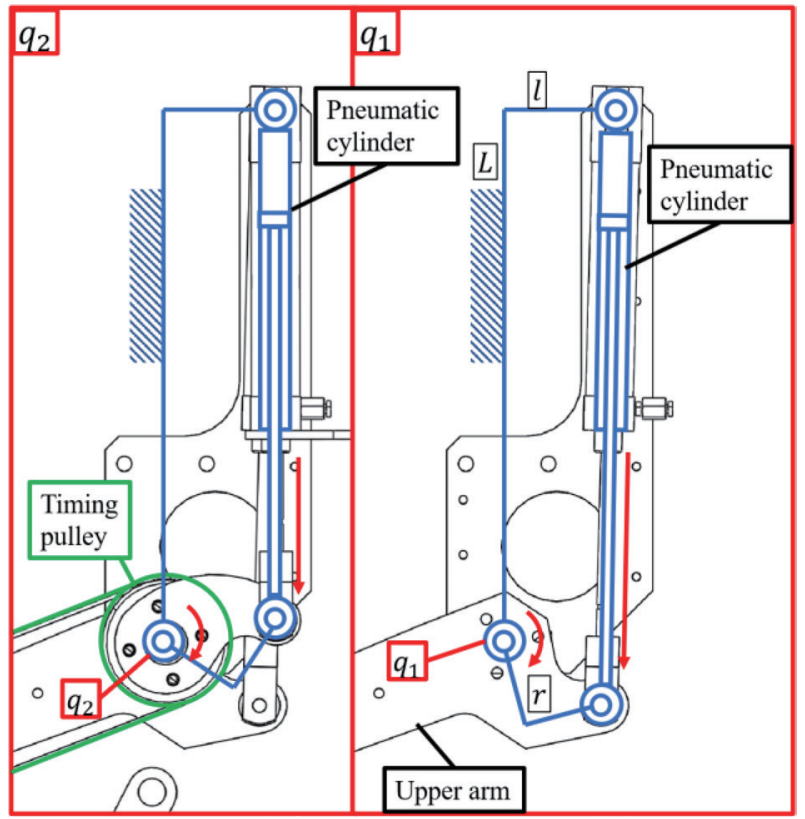

Fig. 4. (Color online) Slider-crank mechanism for driving arm. Left: mechanism of joint $\mathrm{J}_{2}$. The pneumatic cylinder rotates the timing pulley through the mechanism. Right: mechanism of joint $\mathrm{J}_{1}$. The pneumatic cylinder rotates the upper arm.

mounted as shown in Fig. 5. The upper arm has an oscillating mechanism with a gas spring. The forearm is raised by two springs mounted on the arm driving unit. The torque generated by the gravity compensation mechanism is

$$
\tau_{g c}=\left[\begin{array}{c}
\tau_{g c 1} \\
\tau_{g c 2}
\end{array}\right]=\left[\begin{array}{c}
\frac{a b C_{1} f_{g s}}{\sqrt{a^{2}+b^{2}+2 a b S_{1}}} \\
k_{s} J_{a 2}\left(d_{2}-d_{n}\right)
\end{array}\right] .
$$




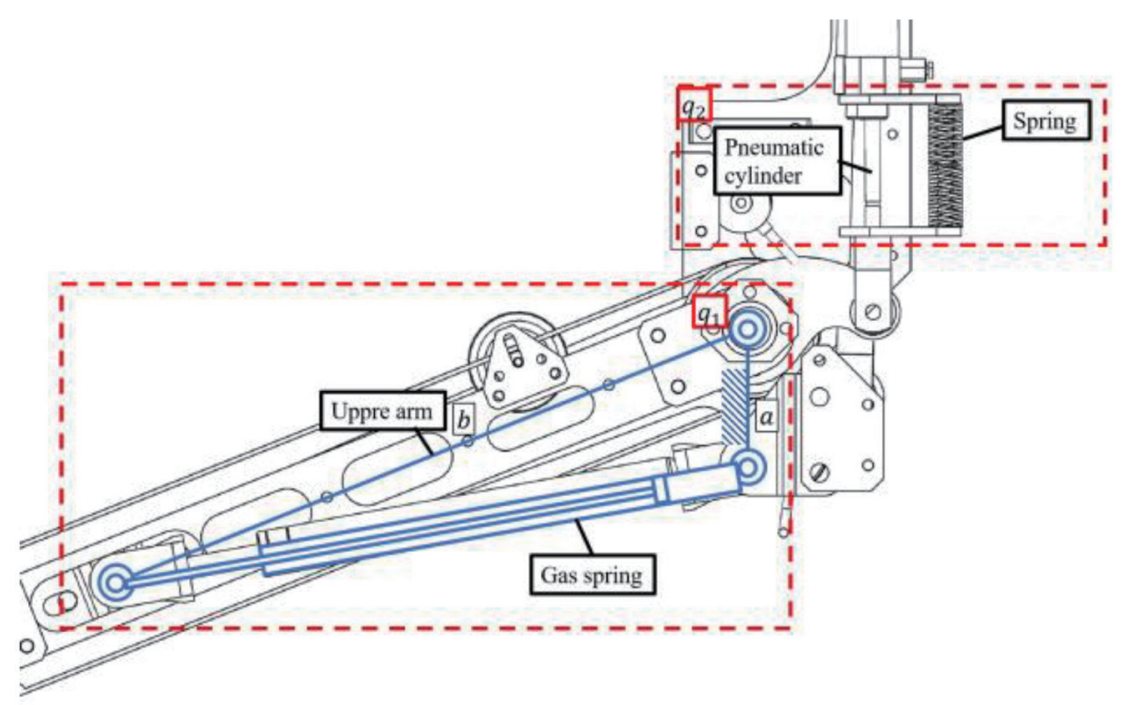

Fig. 5. (Color online) Gravity compensation mechanism.

The wrist driving unit, shown in Fig. 6, has two vane motors and a pneumatic cylinder. The vane motor that drives joint $J_{3}$ rotates the forearm around an axis along the forearm. The pneumatic cylinder rotates joint $\mathrm{J}_{4}$ through the slider-crank mechanism. The relationship between the joint torque and the driving force is given by Eq. (11). The actuators of joints $\mathrm{J}_{3}$ and $q_{4}$ are placed inside the forearm and are close to joint $\mathrm{J}_{2}$. Therefore, the torque of joint $q_{2}$ is less affected by the gravity force of the actuators. The vane motor that drives joint $\mathrm{J}_{5}$ is mounted on the tip of the wrist and it rotates the forceps around the roll axis.

\subsection{Kinematic model of the virtual RCM}

The kinematic model of the RCM is required to move the instruments around the RCM. Figure 7 shows the kinematic model of the RCM. The kinematics is as follows:

$$
\begin{aligned}
& \boldsymbol{P}_{o p}=R_{o p}\left[\begin{array}{c}
0 \\
D \\
0
\end{array}\right], \\
& R_{o p}=R_{z} R_{y} R_{x} .
\end{aligned}
$$

The reference position and reference rotation matrix of the robot are respectively obtained as

$$
\begin{gathered}
\boldsymbol{P}_{\boldsymbol{t}}=\boldsymbol{P}_{\boldsymbol{o p}}+\boldsymbol{P}_{\boldsymbol{r c m}}, \\
R_{t}=R_{o p} .
\end{gathered}
$$




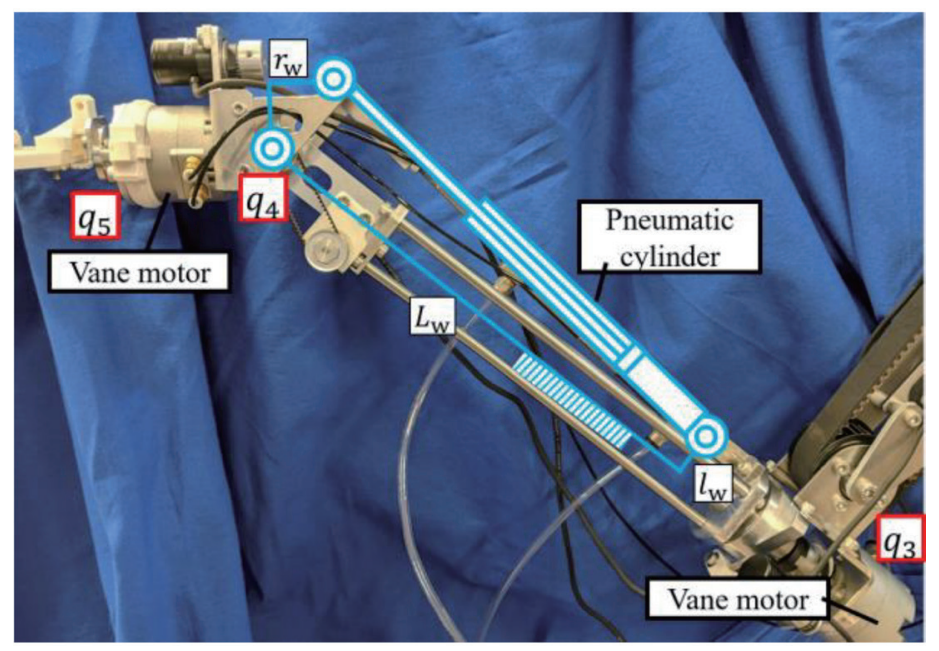

Fig. 6. (Color online) Wrist driving unit.

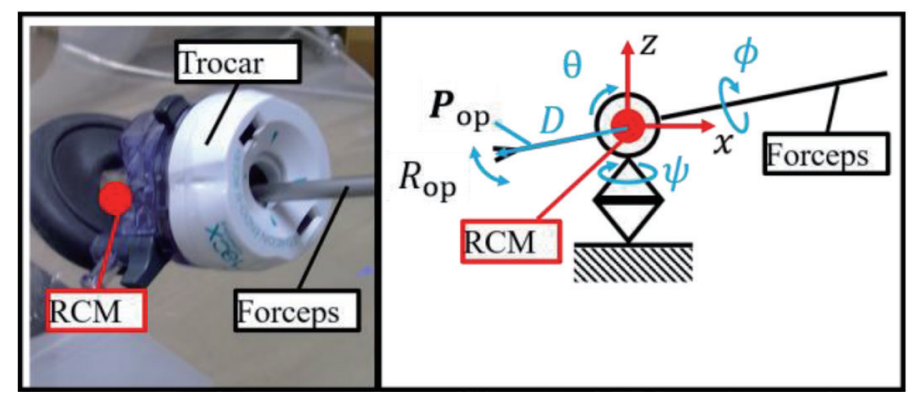

Fig. 7. (Color online) Kinematic model of the RCM.

The position of the RCM is set by the following step when the forceps are inserted into the trocar. First, the operator moves the robotic arm passively and adjusts the position of the forceps tip such that it coincides with the position of the RCM. Next, the operator pushes a button on the user interface, and then the position of the forceps tip is calculated using Eq. (1) and registered as the position of the RCM.

\section{Pneumatic Control System}

\subsection{Position feedback control}

The joints are controlled by the pneumatic servo system. The servo system is a cascade control. The main loop is a position feedback loop and the minor loop is the driving force or torque feedback loop. The controllers of the main loop and minor loop are a 
proportional-derivative (PD) controller and a proportional-integral (PI) controller, respectively. Figure 8 shows a block diagram of the position control system and Table 1 shows the PID gains. The $\mathrm{PD}$ gain of joint $\mathrm{J}_{0}$ is the variable gain proposed in Sect. 3.3.

\subsection{Gravity compensation}

The torque generated by the gravity force of the arm impairs the performance of the position control system. This gravity force is compensated by feedforward compensation of the reference joint torques $\tau_{1}$ and $\tau_{2}$ to improve the performance. The arm gravity compensation is expressed as

$$
\tau_{g a}=\tau_{g}+\tau_{g c}
$$

The joint torque that should be compensated is increased if instruments are installed in the end effector. The joint torque is given as

$$
\tau_{\boldsymbol{e}}=\left[\begin{array}{c}
\tau_{1} \\
\tau_{2} \\
\tau_{3} \\
\tau_{4}
\end{array}\right]=m g\left[\begin{array}{c}
L_{1} C_{1} \\
L_{2} C_{2}+l_{2} S_{2}+L_{t g}\left(C_{2} C_{4}-S_{2} C_{3} S_{4}\right) \\
-L_{t g} C_{2} S_{3} S_{4} \\
L_{t g}\left(-S_{2} S_{3}+C_{2} C_{3} C_{4}\right)
\end{array}\right],
$$

where $m$ is the mass of the forceps and $L_{t g}$ is the length between the wrist and the center of gravity. The torque $\tau_{e}$ after the instruments are installed is added to the right side of Eq. (18).

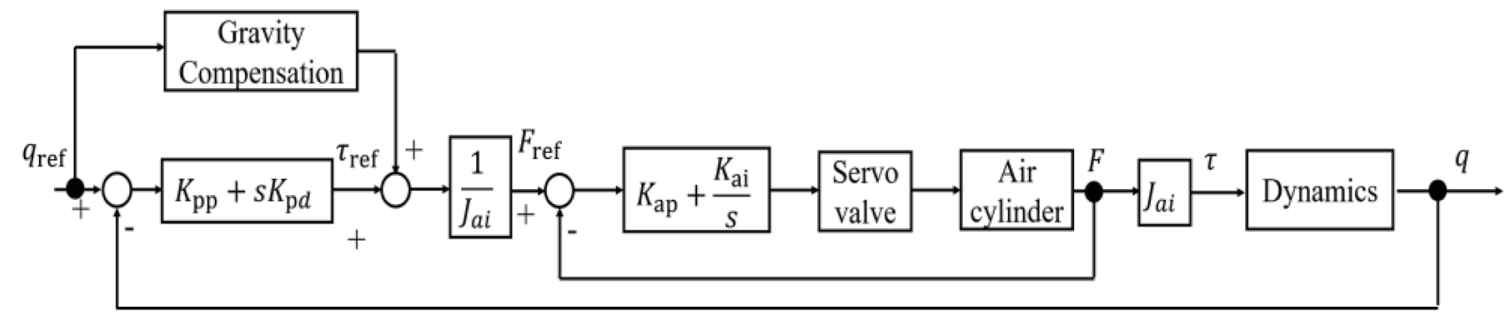

Fig. 8. Block diagram of the pneumatic servo system.

Table 1

PID gains of the position control system.

\begin{tabular}{lcccccc}
\hline Gain & $q_{0}$ & $q_{1}$ & $q_{2}$ & $q_{3}$ & $q_{4}$ & $q_{5}$ \\
\hline$K_{p p}(\mathrm{~N} / \mathrm{rad})$ & Variable gain & 27.0 & 18.0 & 3.20 & 4.20 & 2.50 \\
$K_{p d}(\mathrm{Ns} / \mathrm{rad})$ & Variable gain & 0.80 & 0.40 & 0.07 & 0.03 & 0.03 \\
$K_{a p}(\mathrm{~V} / \mathrm{N}$ or $\mathrm{V} / \mathrm{Nm})$ & 3.30 & 0.04 & 0.03 & 4.00 & 0.15 & 3.00 \\
$K_{a i}(\mathrm{~V} / \mathrm{sN}$ or $\mathrm{V} / \mathrm{sNm})$ & 0.0 & 1.00 & 1.00 & 5.00 & 1.00 & 1.00 \\
\hline
\end{tabular}




\subsection{Yaw joint control system}

The inertia of joint $\mathrm{J}_{0}$ depends on joint angles $q_{1}$ and $q_{2}$. In the electric servo system, a reduction mechanism decreases the effect of the inertia. In the pneumatic servo system, the inertia affects the control system because no reduction mechanism is mounted. The PD gains should be large for precise position control. However, large gains make the system unstable when the arms are folded. To decrease the effect of the inertia, a method of varying the gain with the joint angles is implemented. According to the dynamic model of the pneumatic actuator, ${ }^{(20-22)}$ the relationship between the driving torque and the input voltage of the valve is

$$
\begin{gathered}
\tau_{0}=\frac{K_{a} A\left(u-s K_{b} q_{0}\right)}{s}, \\
K_{a}=K_{s v} P_{s} \sqrt{R T_{a}}(1+\delta) A, \\
K_{b}=\frac{A^{2} P_{o}}{V_{o}},
\end{gathered}
$$

where $s$ is the Laplace operator, $K_{s v}$ is the ratio between the flow rate and $u, P_{S}$ is the supply pressure, $R$ is the gas constant, $T_{a}$ is the temperature of the air, and $P_{o}$ and $V_{o}$ are the pressure and volume of the vane motor at the equilibrium state, respectively. Equation (20) indicates that the angular velocity affects the driving torque. To compensate for the effect, the following voltage is input to the valve:

$$
u=K_{a p}\left(\tau_{0 r e f}-\tau_{0}\right)+s K_{b} q_{0}
$$

The value of $K_{b}=0.24 \mathrm{Ns} / \mathrm{rad}$ was determined by trial and error. The position control system is given as

$$
\frac{q_{0}}{q_{0 r e f}}=\frac{K_{a} A\left(K_{a p}+\frac{K_{a i}}{s}\right)\left(K_{p p}+s K_{p d}\right)}{s(I s+B)\left(s+K_{a} A\right)\left(K_{a p}+\frac{K_{a i}}{s}\right)+K_{a} A\left(K_{a p}+\frac{K_{a i}}{s}\right)\left(K_{p p}+s K_{p d}\right)} .
$$

$K_{a}$ is large because $P_{s}=600(\mathrm{kPa})$. Assuming that $K_{a}$ is large, the term of $s^{3}$ can be ignored, giving the following second-order system:

$$
\frac{q_{0}}{q_{0 r e f}}=\frac{K_{p p}+s K_{p d}}{I s^{2}+\left(B+K_{p d}\right) s+K_{p p}} .
$$


The inertia around the yaw axis is given as

$$
\begin{aligned}
I\left(q_{1}, q_{2}\right)= & I_{0 z}+R_{1}\left[\begin{array}{ccc}
I_{1 x} & I_{1 x y} & I_{1 x z} \\
I_{1 y x} & I_{1 y} & I_{1 y z} \\
I_{1 z x} & I_{1 z y} & I_{1 z}
\end{array}\right] \boldsymbol{R}_{1}^{\boldsymbol{t}}+R_{2}\left[\begin{array}{ccc}
I_{2 x} & I_{2 x y} & I_{2 x z} \\
I_{2 y x} & I_{2 y} & I_{2 y z} \\
I_{2 z x} & I_{2 z y} & I_{2 z}
\end{array}\right] \boldsymbol{R}_{2}^{\boldsymbol{t}} \\
& +M_{1} L_{1 g}{ }^{2} C_{1}^{2}+M_{2}\left(L_{1} C_{1}+L_{1 g} C_{2}+l_{2 g} S_{2}\right)^{2} \\
= & I_{0 z}+I_{1 y} S_{1}^{2}+\left(I_{1 z}+M_{1} L_{1 g}{ }^{2}\right) C_{1}^{2}-2 I_{1 y z} S_{1} C_{1}+I_{2 y} S_{2}^{2} \\
& +I_{1 z} C_{2}{ }^{2}-2 I_{2 y z} S_{2} C_{2}+M_{2}\left(L_{1} C_{1}+L_{1 g} C_{2}+l_{2 g} S_{2}\right)^{2} .
\end{aligned}
$$

The inertia can be calculated using Eq. (26) after measuring the joint angles.

The variable PD gain of the position feedback loop is

$$
\begin{gathered}
K_{p p}\left(q_{1}, q_{2}\right)=\omega_{n}^{2} I\left(q_{1}, q_{2}\right), \\
K_{p d}\left(q_{1}, q_{2}\right)=2 \sqrt{I\left(q_{1}, q_{2}\right) K_{p p}} \zeta-B .
\end{gathered}
$$

The variable PD gains $K_{p p}\left(q_{1}, q_{2}\right)$ and $K_{p d}\left(q_{1}, q_{2}\right)$ depend on the joint angles.

By substituting $K_{p p}\left(q_{1}, q_{2}\right)$ and $K_{p d}\left(q_{1}, q_{2}\right)$ of Eqs. (27) and (28) into Eq. (25), the characteristic equation Eq. (25) becomes the equation of the second-order system. The robotic arm's dynamic parameters $\omega_{n}$ and $\zeta$ and controller gains should be determined to make the second-order system given by Eq. (25) stable.

\section{Experiments}

\subsection{Inertia estimation}

To estimate the inertia of the arms, an inertia estimation experiment was conducted. The purpose of this experiment was to estimate the inertias of the forearm and upper arm, $I_{0 z}, I_{1 y}, I_{1 z}$, $I_{1 y z}, I_{2 z}, I_{2 y}$, and $I_{2 y z}$ and the viscosity $B$ of joint $\mathrm{J}_{0}$. The inertias $I_{0 z}, I_{1 y}, I_{1 z}, I_{1 y z}, I_{2 z}, I_{2 y}$, and $I_{2 y z}$ were estimated using Eq. (27) with some values of $I\left(q_{1}, q_{2}\right)$ measured in the experiment. The inertia $I\left(q_{1}, q_{2}\right)$ can be calculated using Eq. (26) if $K_{p p}$ is fixed and $\omega_{n}$ is estimated. The natural frequency $\omega_{n}$ was estimated by inputting the step input into joint $\mathrm{J}_{0}$. In this experiment, the step input was input to the yaw angle with various values of joint angles $q_{1}$ and $q_{2}$. The PD gains of joint $\mathrm{J}_{0}$ were set as $K_{p p}=16 \mathrm{Nm} / \mathrm{rad}$ and $K_{p d}=0.3 \mathrm{Nms} / \mathrm{rad}$, and the gains were determined to control the joint angle $q_{0}$ as quickly as possible when the arm was in the folding position with joint angle $q_{1}$ of $0^{\circ}$ and joint angle $q_{2}$ of $60^{\circ}$. First, the reference angle $q_{1}$ was set from -20 to $-90^{\circ}$. Next, $q_{2}$ was set from 65 to $0^{\circ}$. Finally, $q_{1}$ was set from -90 to $-20^{\circ}$. The step of the 
reference joint angle $q_{0}$ was $10^{\circ}$, and the step reference of joint $\mathrm{J}_{0}$ was measured in each step. Figure 9 shows an example of the step response of $q_{0}$. The measured angle indicates the encoder angle and the theoretical angle indicates the theoretical step response of the second-order delay system calculated using the estimated $\omega_{n}$ and $\zeta$. The theoretical step response is given as Eq. (29). The dead time $T_{d}$, peak time $T_{p}$, period $T_{n}$, and 5\% setting time $T_{s}$ were obtained from the step response. The relationship between $\omega_{n}$ and the obtained times and the relationship between $\zeta$ and the obtained times are given by Eqs. (31) and (32), respectively.

$$
\begin{gathered}
q_{0}=Q_{0} e^{-\omega_{n} \sqrt{1-\zeta^{2}}\left(t-T_{d}\right)} \cos \left(\omega_{n} \sqrt{1-\zeta^{2}}\left(t-T_{d}\right)\right), \\
T_{d}=T_{p}-\frac{T_{n}}{2}, \\
\omega_{n}=\sqrt{\left(\frac{4 \pi}{T_{n}}\right)^{2}+\left(\frac{9}{T_{s}-T_{d}}\right)^{2}}, \\
\zeta=\sqrt{1-\frac{2 \pi}{T_{n} \omega_{n}}} .
\end{gathered}
$$

Table 2 shows the estimated inertias.

\subsection{Yaw control experiment}

To confirm that the method of control using variable gain decreases the effect of differences in the inertia, the step responses of $q_{0}$ with the variable gain and fixed gain were compared. The fixed PD gains of joint $\mathrm{J}_{0}$ were set as $K_{p p}=16 \mathrm{Nm} / \mathrm{rad}$ and $K_{p d}=0.3 \mathrm{Nms} / \mathrm{rad}$. The experiments

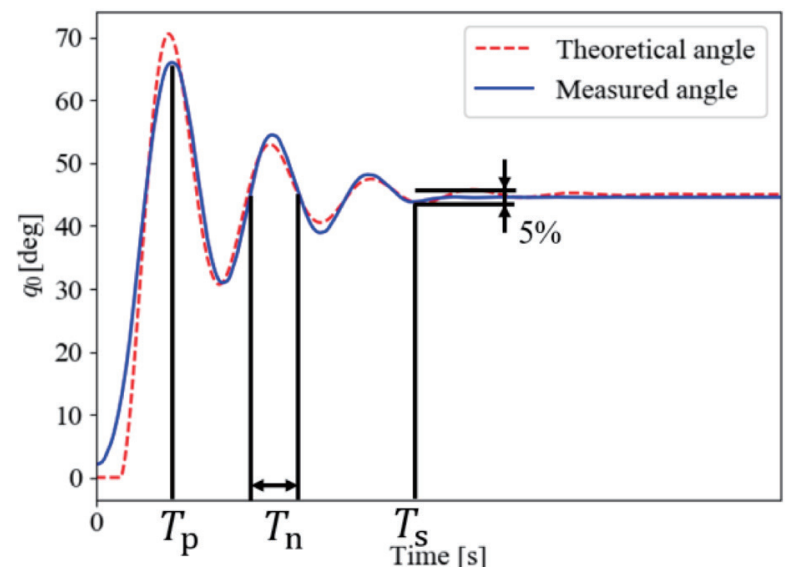

Fig. 9. (Color online) Experimental result of the step response.
Table 2

Estimated inertias of the arm.

\begin{tabular}{lcc}
\hline Parameter & Value & Unit \\
\hline$I_{0 z}$ & 5.89 & $\mathrm{gm}^{2}$ \\
$I_{1 y}$ & 7.35 & $\mathrm{gm}^{2}$ \\
$I_{1 z}$ & 99.1 & $\mathrm{gm}^{2}$ \\
$I_{1 y z}$ & 16.9 & $\mathrm{gm}^{2}$ \\
$I_{2 y}$ & 34.9 & $\mathrm{gm}^{2}$ \\
$I_{2 z}$ & 68.6 & $\mathrm{gm}^{2}$ \\
$I_{2 y z}$ & 35.0 & $\mathrm{gm}^{2}$ \\
$B$ & 0.17 & $\mathrm{Nms} / \mathrm{rad}$ \\
\hline
\end{tabular}


were conducted with four settings of joint angles $q_{1}$ and $q_{2}$, as shown in Fig. 10. Figures 11(a) and 11(b) show the experimental results of the fixed gain and variable gain, respectively. The horizontal and vertical axes indicate the elapsed time and joint angle, respectively. The expected reference is the theoretical transient response of $\omega_{n}=2 \mathrm{~Hz}$ and $\zeta=0.5$. The values of $\omega_{n}$ and $\zeta$ were determined experimentally. Figure 11(a) shows that each response is different. In particular, the response of setting 1 takes a long time to reach the static state. Figure 11(b) shows that most of the responses coincide with the theoretical response. Therefore, the proposed method of control using variable gain decreases the effect of differences in the inertia, improving the performance of the position control.

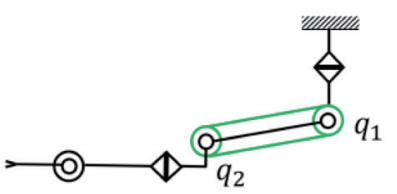

(1) $\left[\begin{array}{ll}q_{1} & q_{2}\end{array}\right]=\left[\begin{array}{ll}-70 & 0\end{array}\right][\mathrm{deg}]$

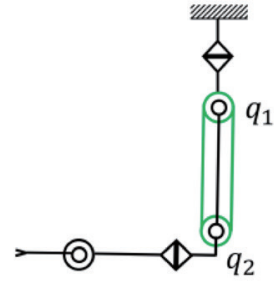

(3) $\left[\begin{array}{ll}q_{1} & q_{2}\end{array}\right]=\left[\begin{array}{ll}0 & 0\end{array}\right][\mathrm{deg}]$

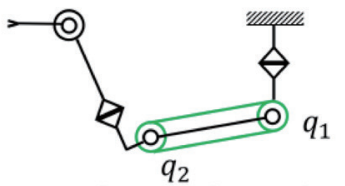

(2) $\left[\begin{array}{ll}q_{1} & q_{2}\end{array}\right]=\left[\begin{array}{ll}-70 & 60\end{array}\right][\mathrm{deg}]$

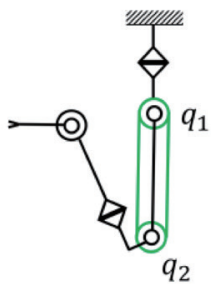

(4) $\left[\begin{array}{ll}q_{1} & q_{2}\end{array}\right]=\left[\begin{array}{ll}0 & 60\end{array}\right][\mathrm{deg}]$

Fig. 10. (Color online) Experimental settings of joint angles $q_{1}$ and $q_{2}$.

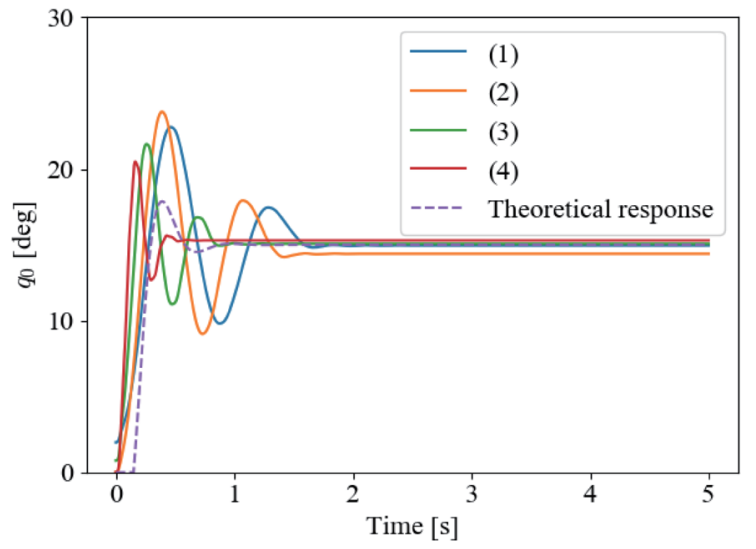

(a)

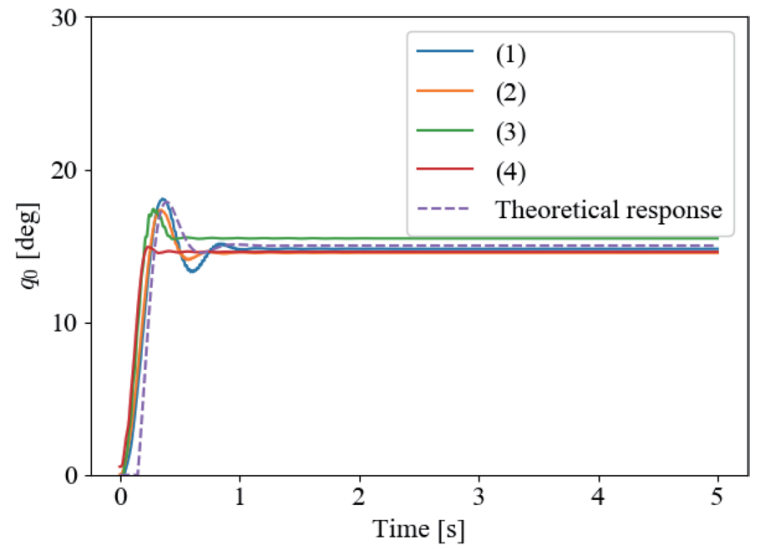

(b)

Fig. 11. (Color online) Comparison between fixed and variable gains. (a) Experimental result of fixed gain. (b) Experimental result of variable gain. 


\subsection{Pivot motion control experiment}

To confirm that the robotic arm can move the instruments by pivoting around the RCM, sinusoidal waves were input into the angles $\psi$ and $\theta$. The reference angles were given by the following sinusoidal wave:

$$
\left[\begin{array}{l}
\psi \\
\theta
\end{array}\right]=\left[\begin{array}{l}
15 \\
15
\end{array}\right] \sin 2 \pi f t(\operatorname{deg}),
$$

where $f=0.2 \mathrm{~Hz}$ is the frequency. The forceps was installed on the end effector and the gravity was compensated. The mass $m=115 \mathrm{~g}$ and the center of gravity $L_{t g}=247 \mathrm{~mm}$ of the forceps were measured beforehand. The position of the RCM was that mentioned in Sect. 2.3. The ground truth of $\psi$ and $\theta$ was calculated from the rotation matrix $R_{t}$ using

$$
\begin{gathered}
\psi=\tan ^{-1}\left(\frac{-\boldsymbol{R}_{\boldsymbol{t}}(1,2)}{\boldsymbol{R}_{\boldsymbol{t}}(2,2)}\right), \\
\theta=\sin ^{-1} \boldsymbol{R}_{\boldsymbol{t}}(3,2) .
\end{gathered}
$$

The experimental results are shown in Fig. 12 and show that the measured angles follow the reference angles. An overshoot of $2^{\circ}$ can be seen in the results of $\psi$. The angle $\psi$ is mainly controlled by joint angles $q_{0}$ and $q_{3}$. The experimental results of $q_{0}$ and $q_{3}$ are shown in Fig. 13 and indicate that whereas $q_{0}$ follows the reference angle, the experimental result of $q_{3}$ has a slight delay. The overshoot of $\psi$ is caused by the delay of $q_{3}$. The delay can be decreased if the friction force of the vane motor is compensated. We confirmed that the robotic arm can move the forceps by pivoting around the RCM.

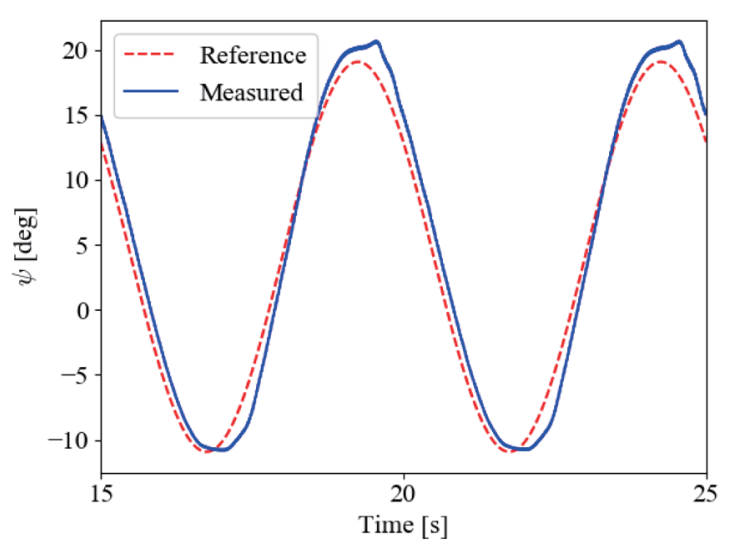

(a)

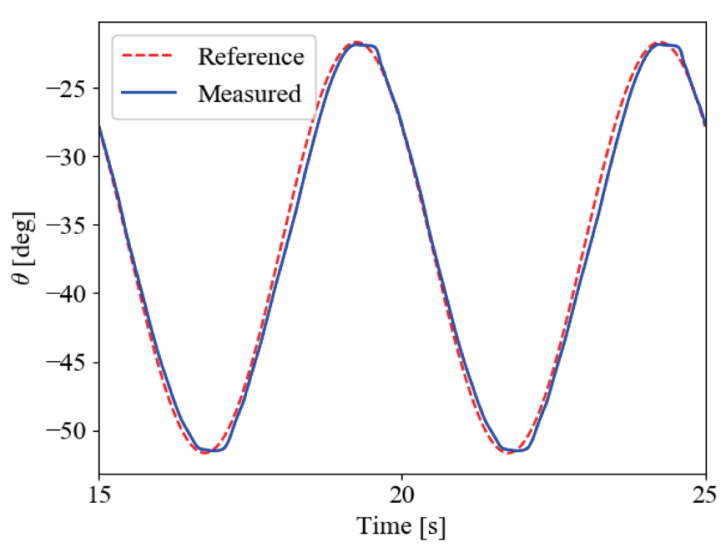

(b)

Fig. 12. (Color online) Results of the pivot motion experiment. (a) Experimental result of $\psi$. (b) Experimental result of $\theta$. 


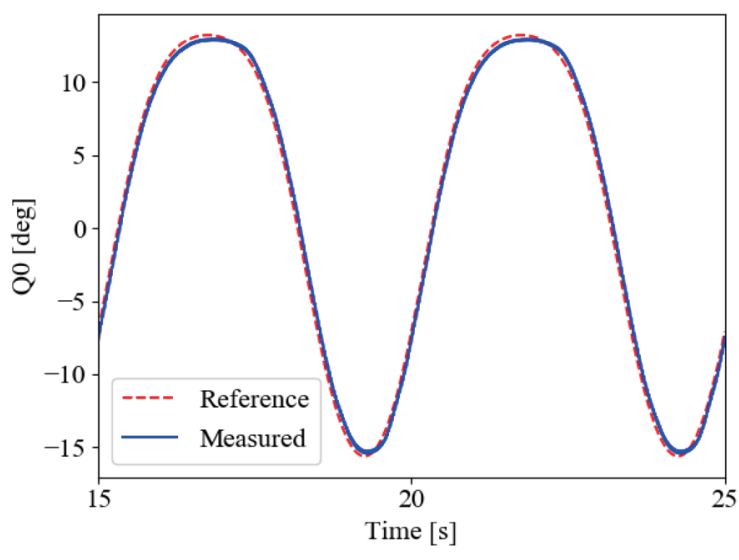

(a)

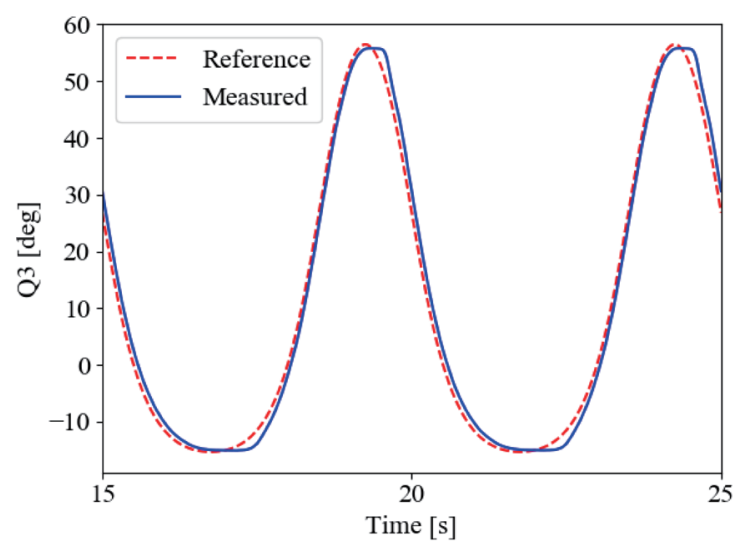

(b)

Fig. 13. (Color online) Experimental results of joint angles $q_{0}$ and $q_{3}$. (a) Experimental result of $q_{0}$. (b) Experimental result of $q_{3}$.

In surgical operation, the velocity of the forceps tip is low, and the angular velocity of the pivot motion can be scaled by the length between the RCM and the tip by controlling the arm posture. Therefore, the robotic arm only needs a low control bandwidth, and the experimental results of Figs. 12 and 13 suggest that the developed robotic arm and its control system have potential use in a practical surgical scene.

\section{Conclusion}

In this study, a pneumatically driven robotic arm for laparoscopic surgery was developed. The mass of the robotic arm is only $4 \mathrm{~kg}$ and the payload at the tip is $1 \mathrm{~kg}$. The pivot motion generated by the kinematic model allows the RCM to be set easily. The driving mechanism makes the robotic arm lightweight and compact. The gravity compensation and the method of varying the PD gain depending on the inertia improved the performance of the position control. As future work, the operability of the robotic arm should be evaluated and the position of the RCM should be detected and registered automatically.

\section{References}

1 R. A. Beasley: J. Rob. 2012 (2012). https://doi.org/10.1155/2012/401613

2 A. Simorov, R. S. Otte, C. M. Kopietz, and D. Oleynikov: Surg. Endosc. 26 (2012) 2117. https://doi.org/10.1007/ s00464-012-2182-y

3 S. W. Kang, S. C. Lee, S. H. Lee, K. Y. Lee, J. J. Jeong, Y. S. Lee, K. H. Nam, H. S. Chang, W. Y. Chung, and C. S. Park: Surgery 146 (2009) 1048. https://doi.org/10.1016/j.surg.2009.09.007

4 R. H. Taylor and D. Stioinanovici: IEEE Trans. Rob. Autom. 19 (2003) 765. https://doi.org/10.1109/ TRA.2003.817058

5 R. Rao, R. Nayyar, S. Panda, and A. K. Hemal: J. Rob. Surg. 1 (2007) 217. https://doi.org/10.1007/s11701-0070030-1

6 J. H. Kaouk, G. P. Haber, R. Autorino, S. Crouzet, A. Ouzzane, V. Flamand, and A. Villers: Eur. Urol. 66 (2014) 1033. https://doi.org/10.1016/j.eururo.2014.06.039 
7 C. Gosrisirikul, K. D. Chang, A. A. Raheem, and K. H. Rha: Asian J. Endosc. Surg. 11 (2018) 291. https://doi. org/10.1111/ases.12660

8 J. E. N. Jaspers, M. Bentala, J. L. Herder, B. A. de Mol, and C. A. Grimbergen: Minm. Invasive Ther. Allied Technol. 13 (2004) 191. https://doi.org/10.1080/13645700410032851

9 B. Hannaford, J. Rosen, D. W. Friedman, H. King, P. Roan, L. Cheng, D. Glozman, J. Ma, S. N. Kosari, and L. White: IEEE Trans. Biomed. Eng. 60 (2013) 954. https://doi.org/10.1109/TBME.2012.2228858

10 D. Stephan, H. Sälzer, and F. Willeke: Telerobotic Syst. Visceral Surg. 34 (2018) 31. https://doi. org/10.1159/000486111

11 U. Kim, D. Seok, Y. B. Kim, D. Lee, and H. R. Choi: Proc. IEEE/RSJ Int. Conf. Intelligent Robots and Systems (IEEE/RSJ, 2016) 845-850. https://doi.org/10.1109/IROS.2016.7759149

12 Y. Tsukamoto and C. Ishii: Proc. Int. Conf. Robotics and Biomimetics (IEEE, 2014) 1650-1655. https://doi. org/10.1109/ROBIO.2014.7090571

13 J. J. Rassweiler, R. Autorino, J. Klein, A. Mottrie, A. S. Goezen, J. U. Stolzenburg, K. H. Rha, M. Schurr, J. Kaouk, V. Patel, P. Dasgupta, and E. Liatsikos: J. British Assoc. Urological Surg. 120 (2017) 822. https://doi. org/10.1111/bju.13851

14 L. J. M. van den Bedem: Realization of a Demonstrator Slave for Robotic Minimally Invasive Surgery, Ph.D. thesis, Technische Universiteit Eindhoven (2010). https://doi.org/10.6100/IR684835

15 M. J. Maurice, D. Ramirez, and J. H. Kaouk: Eur. Urol. 71 (2017) 643. https://doi.org/10.1016/j.eururo.2016.06.005

16 U. Hagn, R. Konietschke, A. Tobergte, M. Nickl, S. Jörg, B. Kübler, G. Passig, M. Gröger, F. Fröhlich, U. Seibold, L. Le-Tien, A. Albu-Schäffer, A. Nothhelfer, F. Hacker, M. Grebenstein, and G. Hirzinger: Int. J. Comput. Assist. Radiol. Surg. 5 (2010) 183. https://doi.org/10.1007/s11548-009-0372-4

17 D. Haraguchi, T. Kanno, K. Tadano, and K. Kawashima: IEEE/ASME Trans. Mechatron. 20 (2015) 2950. https://doi.org/10.1109/TMECH.2015.2415838

18 K. Takikawa, R. Miyazaki, T. Kanno, G. Endo, and K. Kawashima: J. Rob. Mechatron. 28 (2016) 559. https:// doi.org/10.20965/jrm.2016.p0559

19 K. Tadano, K. Kawashima, K. Kojima, and N. Tanaka: J. Rob. Mechatron. 22 (2010) 179. https://doi. org/10.20965/jrm.2010.p0179

20 K. Kawashima, T. Arai, K. Tadano, T. Fujita, and T. Kagawa: Precis. Eng. 34 (2010) 526. https://doi. org/10.1016/j.precisioneng.2010.02.005

21 S. Liu and J. E. Bobrow: J. Dyn. Syst. Meas. Control 110 (1988) 228. https://doi.org/10.1115/1.3152676

22 T. Iwai, T. Kanno, T. Miyazaki, D. Haraguchi, and K. Kawashima: Int. J. Med. Robot. 16 (2020). https://doi. org/10.1002/rcs.2051

\section{About the Authors}

Takuya Iwai received his M.S. degree in engineering from the Institute of Biomaterials and Bioengineering, Tokyo Medical and Dental University, Tokyo, Japan, in 2018. He is currently pursuing a Ph.D. degree in biomedical sciences and engineering at Tokyo Medical and Dental University. (tiwai.bmc@tmd.ac.jp)

Tetsuro Miyazaki received his Ph.D. degree in engineering from the Department of Mechanical Sciences and Engineering, Tokyo Institute of Technology, in 2014. From 2014 to 2017, he worked as a research assistant (from 2014 to 2015) and an assistant professor (from 2015 to 2017) at Yokohama National University. From 2017 to 2020, he worked as an assistant professor at Tokyo Medical and Dental University. Since 2020, he has been an assistant professor at the Graduate School of Information Science and Technology, The University of Tokyo. He is a member of IEEE. His research interests include mechanical engineering, control engineering, power assistive devices, and medical welfare robotics. (Tetsuro_Miyazaki@ipc.i.u-tokyo.ac.jp) 
Toshihiro Kawase received his B.S., M.S., and Ph.D. degrees from Tokyo Institute of Technology, Tokyo, Japan, in 2007, 2009, and 2012, respectively. He was a research fellow with the Research Institute of National Rehabilitation Center for Persons with Disabilities from 2012 to 2015, and worked as a postdoctoral fellow and a specially appointed assistant professor at Tokyo Institute of Technology from 2015 to 2017. He is currently an assistant professor with Tokyo Medical and Dental University and Tokyo Institute of Technology. He is a member of IEEE. His research interests include medical robots, rehabilitation robotics, and biological signal processing. (kawase.t.aa@m.titech.ac.jp)

Takahiro Kanno received his Ph.D. degree in engineering from the Department of Mechanical Engineering and Science, Kyoto University, in 2013. In 2013, he worked as a postdoctoral researcher at the Precision and Intelligence Laboratory, Tokyo Institute of Technology. From 2013 to 2019, he worked as an assistant professor at Tokyo Medical and Dental University, where he has been an associate professor since 2019. Since 2020, he has been the chief technology officer (CTO) at Riverfield Inc. He is a member of IEEE. His research interests include medical robotics, control engineering, and teleoperation. (t-kanno.rfc@riverfieldinc.com)

Kenji Kawashima received his Ph.D. degree in engineering from the Department of Control Engineering, Tokyo Institute of Technology, in 1997. From 1997 to 2000, he worked as a research assistant at Tokyo Metropolitan College of Industrial Technology. He then worked as an associate professor at the Precision and Intelligence Laboratory, Tokyo Institute of Technology. From 2013 to 2020, he worked as a professor at Tokyo Medical and Dental University. Since 2020, he has been a professor at The University of Tokyo. He is a member of IEEE. His research interests include medical robotics, control engineering, and fluid measurement and control. (kkawa729@g.ecc.u-tokyo.ac.jp) 